Copyright by the American Geophysical Union. Yang, T., W. A. Davis, and W. L. Stutzman (2009), Fundamental-limit perspectives on ultrawideband antennas, Radio Sci., 44, RS1S91, doi:10.1029/2007RS003799.

RADIO SCIENCE, VOL. 44, RS1S91, doi:10.1029/2007RS003799, 2009

\title{
Fundamental-limit perspectives on ultrawideband antennas
}

\author{
Taeyoung Yang, ${ }^{1}$ William A. Davis, ${ }^{1}$ and Warren L. Stutzman ${ }^{1}$ \\ Received 1 December 2007; revised 5 May 2008; accepted 11 December 2008; published 25 February 2009.
}

[1] The fundamental-limit theory of antennas provides a theoretical limit to assist in the evaluation of antenna performance in terms of antenna size, fractional impedance bandwidth, and gain. The limit is very useful in practice, giving a basis for restricting the design search to a class of realizable antennas based on size and performance. Previous research on the limit theory focused on electrically small, resonant antennas.

In this paper, we discuss how the classical fundamental-limit theory can be interpreted for ultrawideband antennas. The frequency response of Chu's equivalent circuit model for spherical modes suggests the concept of an ideal antenna. The transfer function of the ideal antenna, showing ultrawideband antenna characteristics, simply has an entire function and two complex poles. In this paper, an antenna design strategy is developed based on observations of ideal antenna characteristics. A process is presented for evaluating how well an ultrawideband antenna can approach the theoretical size limit based on the 3-dB cutoff frequencies of spherical modes.

Citation: Yang, T., W. A. Davis, and W. L. Stutzman (2009), Fundamental-limit perspectives on ultrawideband antennas, Radio Sci., 44, RS1S91, doi:10.1029/2007RS003799.

\section{Introduction}

[2] Wheeler [1947] first introduced the concept of fundamental-limit theory on antennas. Since his initial work, there have been many investigations into the theoretical limitation of antenna performance versus size [Chu, 1948; Collin and Rothschild, 1964; McLean, 1996; Grimes and Grimes, 1999; Davis et al., 2007]. Over the past half century, researchers have addressed the accuracy and restrictions of the limit theory. The essence of the fundamental-limit theory for antennas creates a trade-off between the size, efficiency, and bandwidth in the design process, and often only two of these factors can be controlled simultaneously within the constraints of the limits.

[3] Although classic limit theories are not necessarily restricted to electrically small antennas, previous research efforts on the limit theory focused on electrically small antennas. Radiation $Q$ (the ratio of total nonradiating energy to average radiated power per radian) has been used to quantify how close an antenna can approach the theoretical limit. The concept of $Q$ is appropriate for resonant (or resonated) antennas. The radiation $Q$ of the

\footnotetext{
${ }^{1}$ Virginia Tech Antenna Group, Department of Electrical and Computer Engineering, Virginia Polytechnic Institute and State University, Blacksburg, Virginia, USA.
}

Copyright 2009 by the American Geophysical Union. 0048-6604/09/2007RS003799 electrically small, resonant antenna can be easily estimated using the well-known, inversely proportional relationship between radiation $Q$ and fractional 3-dB impedance bandwidth [Stutzman and Thiele, 1998]. However, the radiation $Q$ estimate for bandwidth is questionable for low- $Q$ antennas, such as ultrawideband antennas, because the upper bound of the impedance bandwidth for an ultrawideband antenna with a tapered feed structure often approaches infinity.

[4] In this paper, we focus on investigating how the classic fundamental-limit theory can be interpreted for ultrawideband antennas and how a theoretical limit on antenna size and the lower bound of operating frequency can be determined. These aspects are considered using both theory and numerical simulations. A bicone antenna is used as an example to demonstrate the concepts.

\section{Concept of Ideal Antenna and an Antenna Design Strategy}

[5] The wave impedance of spherical $\mathrm{TM}_{0 \mathrm{n}}$ modes (normalized by the intrinsic wave impedance $\eta$ ) can be written as follows [Chu, 1948]:

$$
Z_{n}^{T M}(k r)=\frac{1}{\eta} \frac{E_{\theta}^{+}}{H_{\phi}^{+}}=\frac{j\left(\hat{H}_{n}^{(2)}(k r)\right)^{\prime}}{\hat{H}_{n}^{(2)}(k r)}
$$

where $\hat{H}_{n}^{(2)}$ is the Schelkunoff spherical Hankel function of the second kind and $k$ is the wave number. The "+" 


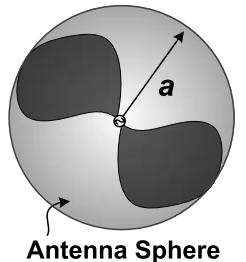

(a)

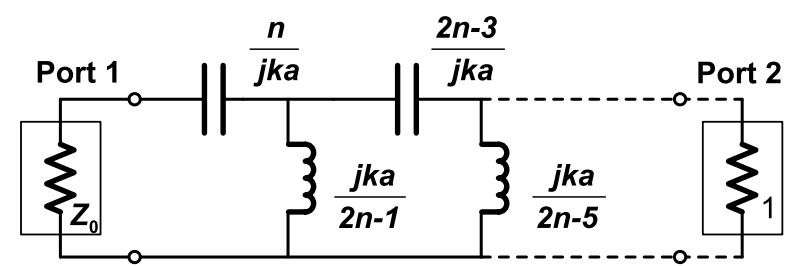

(b)

Figure 1. Model for antenna radiation: (a) Definition of antenna sphere of radius $a$ just enclosing the antenna and (b) Chu's circuit model with two-port scattering parameter representation. A dual ladder network applies to spherical TE modes (used by permission, T. Yang, 2008).

denotes an outgoing wave and the "'," denote a derivative with respect to the argument. Chu's equivalent circuit for the spherical $\mathrm{TM}_{0 \mathrm{n}}$ modes can be found from this normalized wave impedance. In the same manner, the equivalent circuit model for spherical TE cases can also be obtained as a dual to equation (1). In classic fundamental-limit theories, the spherical $\mathrm{TM}_{01}$ mode is emphasized because the spherical $\mathrm{TM}_{01}$ mode provides the minimum radiation $Q$. The higher order modes increase the effective $Q$ of an antenna.

[6] If we consider how much power is delivered to the space beyond the antenna (radiation), Chu's ladder circuit becomes a two-port model as shown in Figure 1. In this case, Port 1 is the antenna sphere circumscribing the antenna structure (see Figure 1a), where $a$ is the radius of the antenna sphere. Port 2 represents the far-field region where radiated power is delivered, though the phase delay, $k r$, associated with the distance is not included in the model. Therefore, $\left|s_{21}\right|^{2}$ represents the transfer of power from the antenna sphere to the radiated far field. For example, $s_{21}$ for the spherical $\mathrm{TM}_{01}$ mode of Chu's model with the two-port scattering parameter representation can be written as

$$
\begin{aligned}
s_{21} & =\frac{2 s^{2}}{2 s^{2}+2 s+1} \\
& =1-0.5\left(\frac{1}{s-(-0.5+j 0.5)}+\frac{1}{s-(-0.5-j 0.5)}\right)
\end{aligned}
$$

where $s=j k a$. This form shows that $s_{21}$ for the fundamental spherical mode consists of an entire function and two complex poles. This represents what we refer to as an ideal antenna, which meets the theoretical performance and size limit. The entire frequency domain and time domain responses of the ideal antenna can be completely described with only two poles that restrict radiation at low frequencies. The ideal antenna has a donut-shaped radiation pattern, with an omni-directional pattern in the azimuth plane. Poleresidue structures of various real antennas were studied extensively by Licul [2004], where the emphasis was on the minimum number of poles needed to reasonable represent the pattern of an antenna over a wide frequency range. Licul showed that a bicone antenna can be characterized in both time and frequency domains with 6 poles. As an exceptional case, large-scale impulseradiating antennas [Baum et al., 1999] may be represented by a simple delta response as an analytic function in an analytical approximation, excluding poles that typically relate to the finite size of the structure.

[7] The pole structure of $s_{21}$ for various spherical TM modes is shown in Figure 2a, with an obvious layering of the poles by modes. These poles offer additional insight into the performance of antenna radiation. The spherical $\mathrm{TM}_{01}$ mode is the simplest mode, with $s_{21}$ determined by the distance between the poles and the complex frequency of interest, plus an entire function. As the mode number $(n)$ increases, the number of poles needed to represent the mode increases. For each mode, the poles with the smallest real part of the complex frequency typically dominate the late-time response.

[8] The corresponding Bode plots of the pole-residue structures for the various spherical modes are shown in Figure 2b. Each spherical TM mode shows the characteristics of a high-pass filter, as modeled by the ladder network form. It is clearly seen that each mode has a cutoff frequency that increases with the mode order $n$. The product of the directivity (given in terms of the $\theta$ and $\phi$ performance of the mode) and $\left|s_{21}\right|^{2}$ for each spherical mode corresponds to the gain of the mode. Therefore, the $\left|s_{21}\right|^{2}$ plot of the Figure 2b shows the variation of gain with frequency. An antenna exciting a specific spherical mode will have a relatively constant gain above the cutoff frequency. This again suggests that the ideal antenna has natural ultrawideband characteristics.

[9] The impulse responses of Chu's equivalent circuits are shown in Figure 2c for various spherical TM modes. The responses correspond to the late-time performance of ultrawideband antennas in the time domain. As the mode number increases, the pulse width narrows. An increase in the size of the antenna sphere $(a)$ results in a wider pulse width, corresponding to a lower cutoff frequency. Because higher-order spherical modes have 


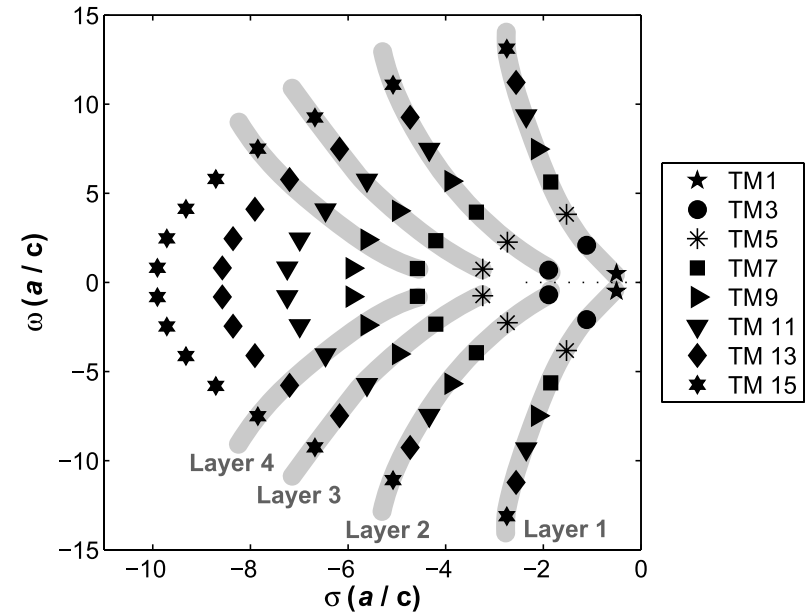

(a)

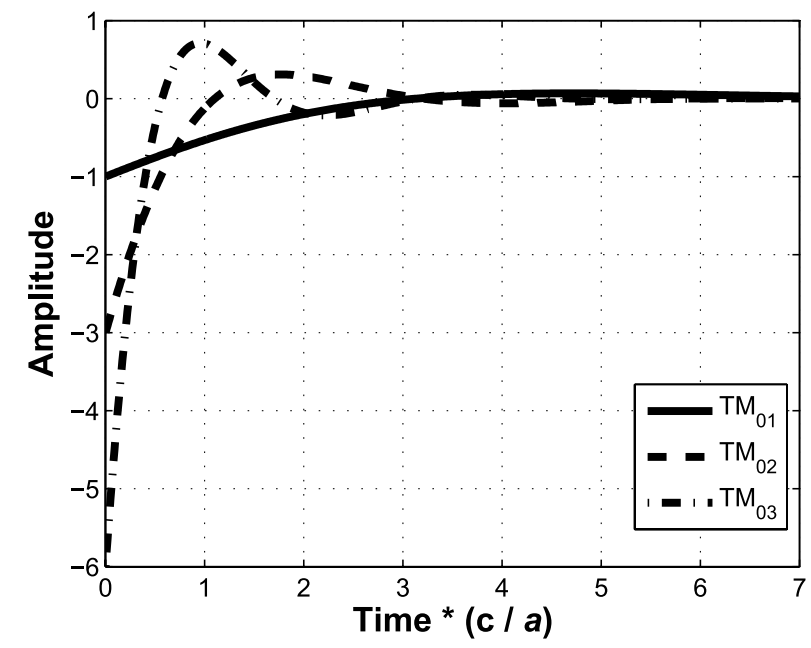

(c)

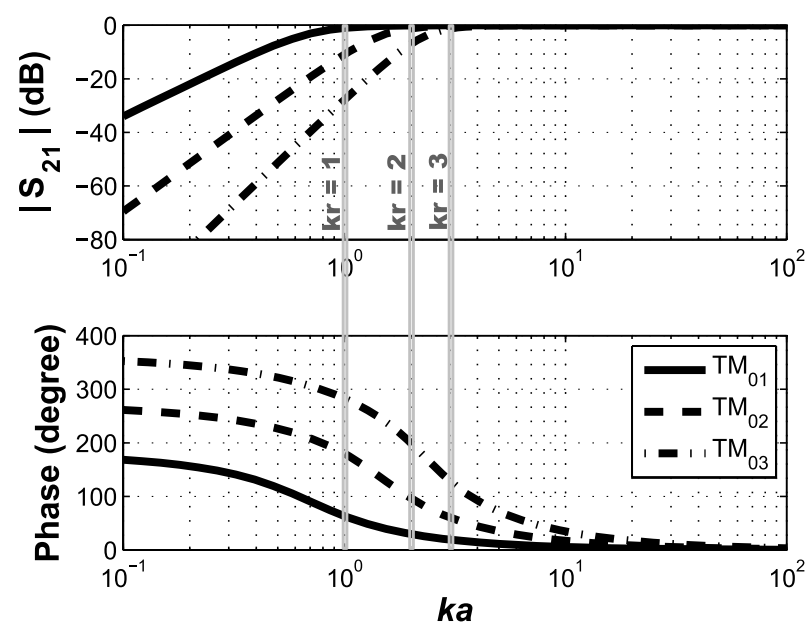

(b)

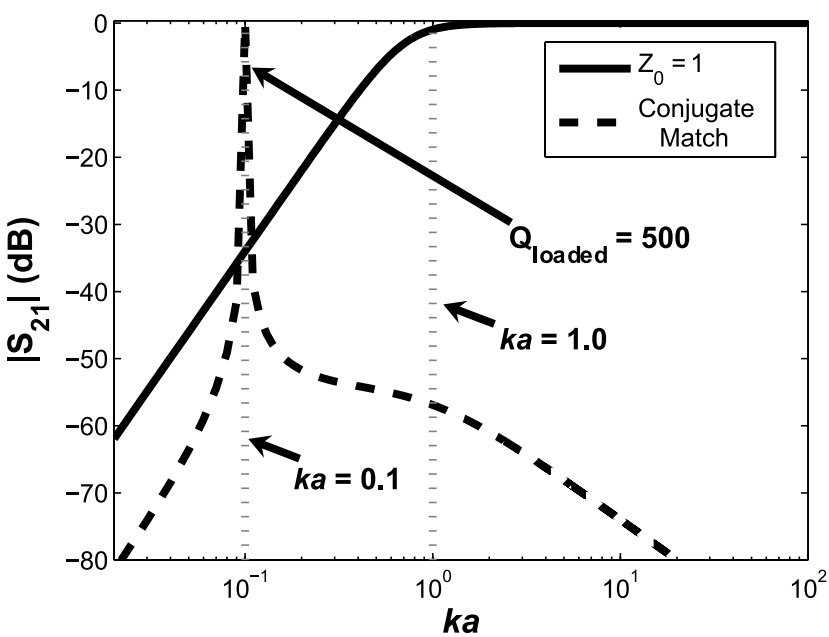

(d)

Figure 2. Characteristics of Chu's circuit model: (a) Pole structure for various spherical $\mathrm{TM}_{0 \mathrm{n}}$ modes in $s$ plane, (b) Bode plot of radiation transfer function $\left|s_{21}\right|$, (c) modal impulse response, and (d) radiation transfer function $\left|s_{21}\right|$ versus electrical size for both large and small $k a$ values (used by permission, T. Yang, 2008).

higher radiation $Q$ values, the resulting late-time responses have some ringing. However, this ringing decays fast.

[10] The concept of the ideal antenna having a high pass characteristic provides insight into antenna design strategies in terms of the trade-off relationship among antenna size, impedance bandwidth, and gain. The strategies are illustrated in Figure $2 \mathrm{~d}$ for the spherical $\mathrm{TM}_{01}$ mode. For a given frequency, the product of wave number $k$ and radius $a$ of the antenna represents the antenna electrical size. If the product $k a$ of an antenna is greater than unity, one may choose the system reference impedance equal to the real part of the input impedance at the antenna sphere in order to maximize the impedance bandwidth of the antenna. For $k a$ smaller than unity (giving an electrically small antenna), the antenna should be tuned to reduce return loss and maximize realized gain. In the example of Figure $2 d$, the antenna was tuned with a conjugate match (dashed curve), such that there is $100 \%$ transmission at the resonant frequency $(k a=0.1)$. However, the impedance bandwidth was limited by the tuning requirement compared to the $k a>1$ case (solid curve).

[11] The achievable impedance bandwidth of the tuned antenna can be found from the classic fundamental-limit theory by using the inverse-proportion relationship 
between the impedance bandwidth and the radiation $Q$. However, as pointed out by Gustafsson and Nordebo [2006], the inverse relationship between radiation $Q$ and impedance bandwidth is only accurate for high- $Q$ antennas $(Q>10)$. It is difficult to obtain a general relationship between the radiation $Q$ and bandwidth. Most recently, Villalobos et al. [2007] investigated theoretical impedance matching limits for ultrawideband antennas based on the Chu's ladder circuits and Fano's theory [Fano, 1950]. Their matching limit provides a guide to optimize the size and bandwidth performance of a designed ultrawideband antenna.

[12] Even though there is an accuracy issue for low- $Q$ antennas, the simple inverse relationship between radiation $Q$ and bandwidth provides a quick insight for antenna engineers. Collin and Rothschild [1964] and McLean [1996] found the radiation $Q$ of the fundamental mode as

$$
Q_{\text {rad }}=\frac{1}{k^{3} a^{3}}+\frac{1}{k a}
$$

This equation has been referred to as the exact form of Chu's limit and is widely accepted in the electromagnetics community. However, Davis [2005] noted an implicit assumption in the derivation of equation (3), which is that energy flows with the speed of light all the way from the antenna sphere to the far-field region. However, the actual phase velocity of the power flow becomes infinity as $k r$ approaches the origin of antenna sphere. This observation suggests that the actual energy velocity varies with distance. Based on this observation, Davis et al. [2007] proposed the following new limit:

$$
Q_{\text {rad }}=\frac{1}{k^{3} a^{3}}
$$

The radiation $Q$ formulas in the classic fundamental-limit theory literature are for unloaded conditions. So, an achievable impedance bandwidth of an antenna with a matched source becomes twice that of the unloaded antenna, a relationship often neglected by researchers.

\section{Size Limitation of Ultrawideband Antennas Illustrated With a Finite-Size Bicone Antenna Example}

[13] In real antenna design problems, the antenna structure acts as an impedance transformer from the system reference impedance $\left(Z_{0}\right.$, typically $\left.50 \Omega\right)$ to the spherical-mode wave impedance. In addition, a designed antenna structure may need to excite a specific spherical mode strongly to have a specific angular coverage (radiation pattern) and range (gain). An antenna can also have additional energy storage mechanisms, represented by additional capacitance and inductance in the circuit model. These additional energy storage mechanisms may increase radiation $Q$, a narrowing of the impedance bandwidth.

[14] A finite-size bicone antenna (see Figure 3a) is a good example to demonstrate how the desired antenna functions can be implemented in a real antenna structure. The bicone antenna is a linearly tapered structure, providing a wideband impedance transformation. To evaluate the performance of the bicone antenna, the antenna of Figure $3 \mathrm{a}$ was characterized with a commercial moment method code [FEKO, 2007]. As shown in Figure 3b, the upper bound of the impedance bandwidth of the bicone antenna is unbounded and is typically limited only by the accuracy details of the feed region. Thus, the bandwidth is extremely large and the conventional radiation $Q$ formula, based on impedance bandwidth, becomes inaccurate and loses meaning for the bicone antenna. The gain versus frequency plot in Figure $3 \mathrm{c}$ shows relatively constant gain over a wide frequency range (pattern is found to also be relatively constant: see Figures $3 \mathrm{e}$ and $3 \mathrm{f}$ ), implying a single spherical mode is strongly excited and dominates the antenna performance. Figure $3 \mathrm{~d}$ shows the radiated $E$ field pulse at $30 \mathrm{~m}$ from the source for a 55 pico second Gaussian input pulse. This radiated $E$ field pulse dictates the antenna transfer function and has a shape of monocycle pulse with slight ringing.

[15] Overall, the bicone antenna is a good ultrawideband antenna. However, we still need to compare the size of the designed bicone antenna to the theoretical limit. The size of an ultrawideband antenna is limited by the lower bound of operating bandwidth as characterized by the excited fundamental mode. Thus, we relate the size of an ultrawideband antenna with the fundamental-mode 3-dB cutoff frequency of $\left|s_{21}\right|^{2}$ shown in Figure $2 \mathrm{~b}$. Note that Chu's circuit model does not consider the actual antenna structure and the connection to the spherical modes.

[16] The spherical-mode model of radiation can be simply represented as the signal-flow diagram of Figure 4a, based on the work of Hansen [1988]. In the model, the relationship between an antenna input port and spherical wave mode is described with scattering parameters representing an antenna transfer function. The excited modes of an arbitrary antenna can be found from measured or simulated near-field data relative to the input port by using spherical mode expansions [Hansen, 1988].

Figure 3. Bicone antenna characteristics: (a) Geometry of finite-size bicone, (b) return loss versus frequency $\left(Z_{0}=\right.$ $100 \Omega$ ), (c) gain versus frequency, (d) transient radiated $E$ field at $30 \mathrm{~m}$ with Gaussian pulse excitation, (e) elevation pattern at $700 \mathrm{MHz}$, and (f) elevation pattern at $7 \mathrm{GHz}$ (used by permission, T. Yang, 2008). 


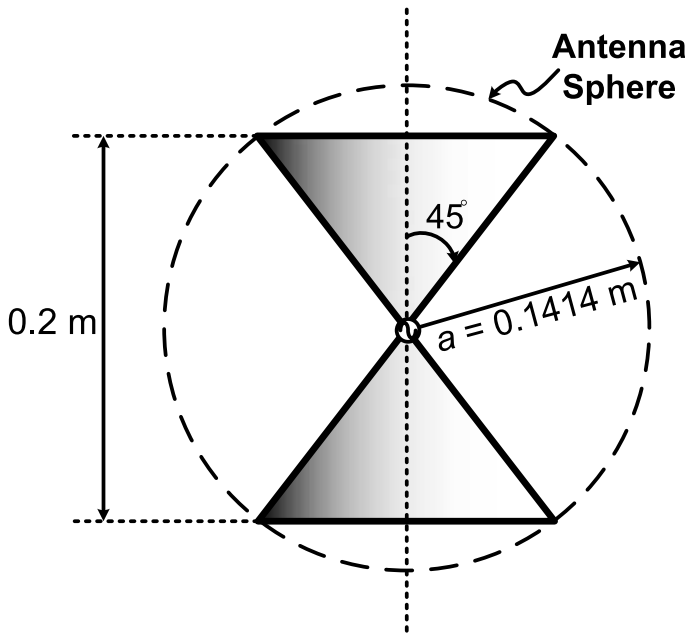

(a)

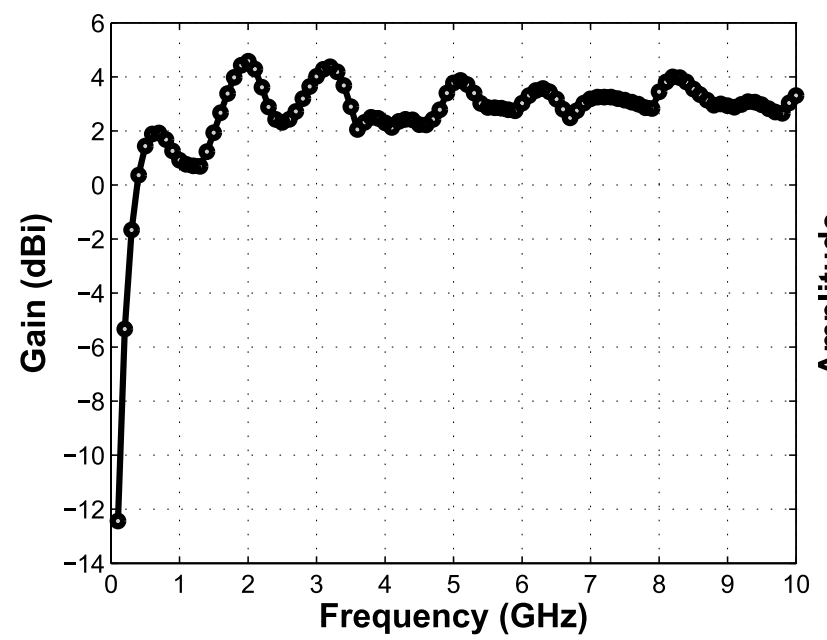

(c)

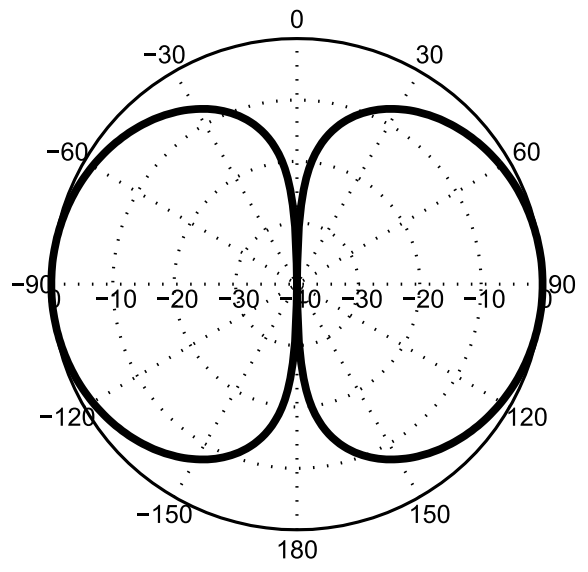

(e)

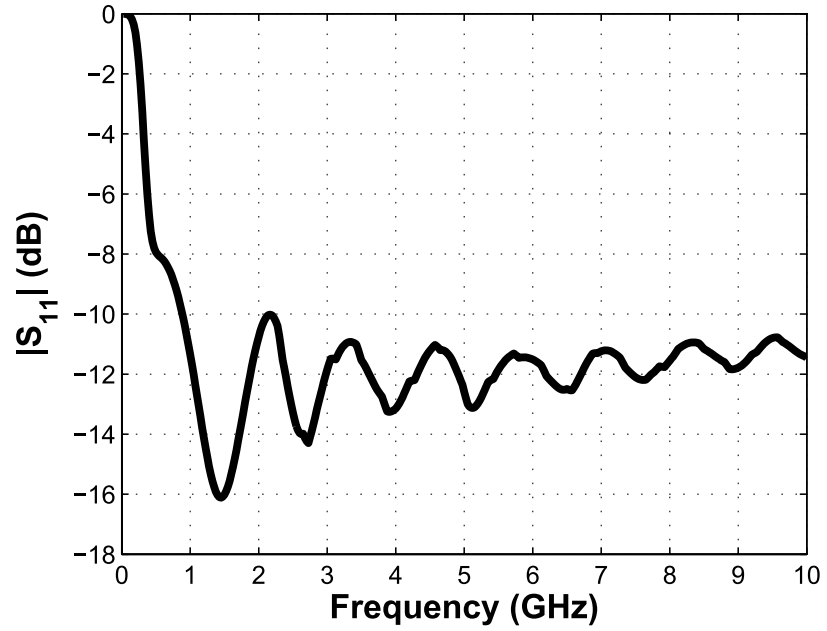

(b)

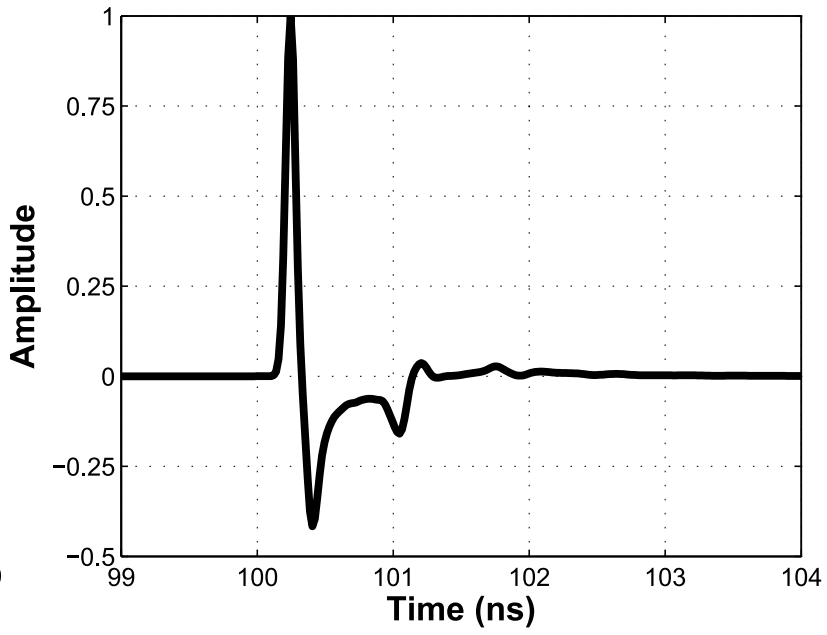

(d)

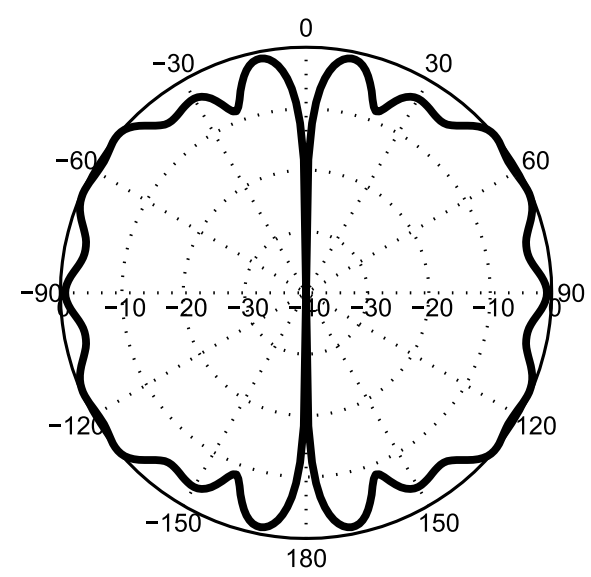

(f)

Figure 3 


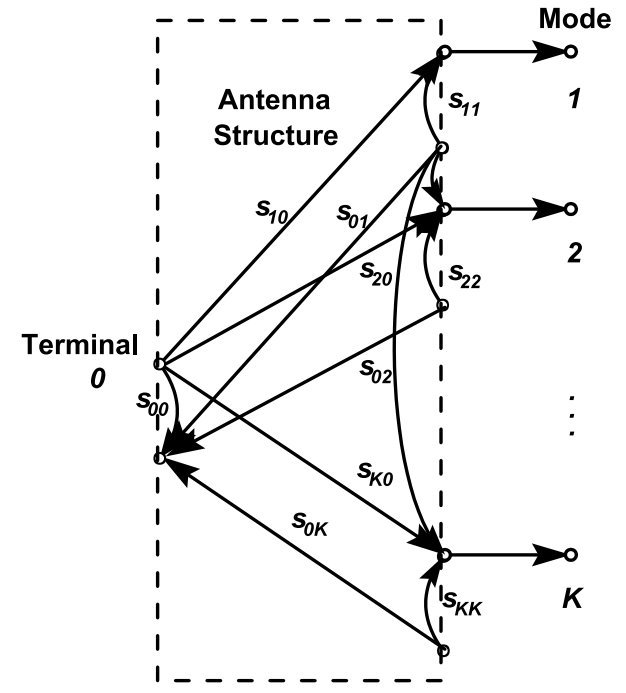

(a)

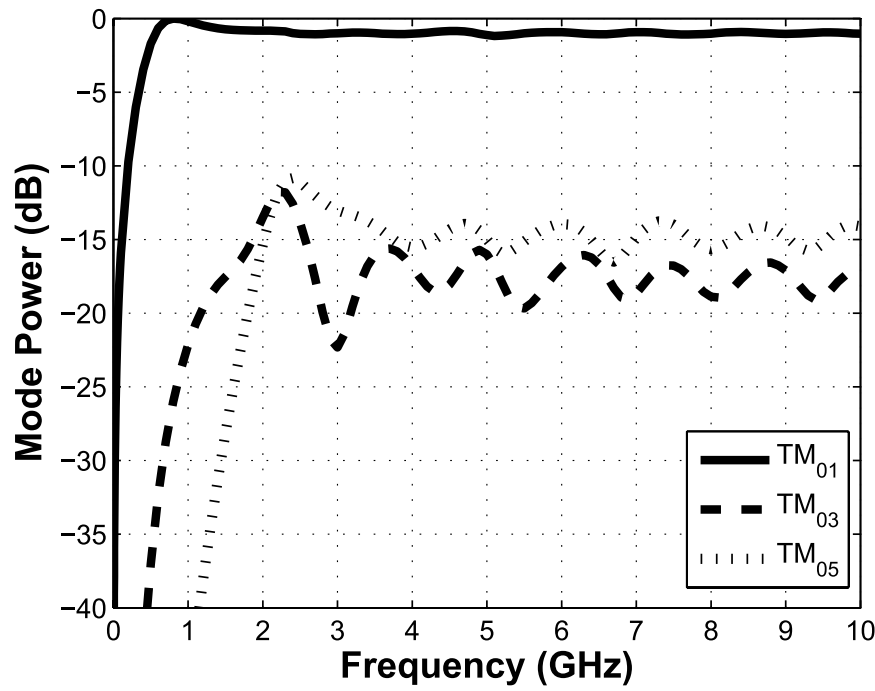

(b)

Figure 4. Antenna modeling of the radiation process: (a) An $S$ parameter representation of radiation coupling to the spherical modes and (b) mode responses of the finite-size bicone (used by permission, T. Yang, 2008).

[17] With an $e^{j \omega t}$ time dependence and outward traveling waves, the spherical vector potentials can be rewritten as

$$
\begin{aligned}
\vec{A} & =\hat{r} \sum_{n=0}^{\infty} \sum_{m=-n}^{n} A_{m, n} \hat{H}_{n}^{(2)}(k r) P_{n}^{|m|}(\cos \theta) e^{j m \phi} \\
\vec{F} & =\hat{r} \sum_{n=0}^{\infty} \sum_{m=-n}^{n} F_{m, n} \hat{H}_{n}^{(2)}(k r) P_{n}^{|m|}(\cos \theta) e^{j m \phi}
\end{aligned}
$$

where $\vec{A}$ and $\vec{F}$ are the magnetic and electric vector potentials, respectively. The coupling coefficients $A_{m, n}$ and $F_{m, n}$ represent the coupling to the $(m, n)$ spherical mode and $P_{n}^{m}(\cos \theta)$ is the associated Legendre function of 1st kind. Using the orthogonal property of the associated Legendre functions, the coupling coefficients can be found from measured or simulated near-field data on the sphere of radius $r_{0}$ as follows:
Then, far-field forms of the electric and magnetic fields contributing to the radiated power can be found from the coupling coefficients as

$$
\begin{aligned}
E_{\theta}(r, \theta, \phi)= & \frac{1}{r} \sum_{m, n}\left[-j \frac{m}{\sin \theta} F_{m n} P_{n}^{|m|}(\cos \theta)\right. \\
& \left.-\eta A_{m n} \frac{d}{d \theta} P_{n}^{|m|}(\cos \theta)\right](j)^{n+1} e^{j(m \phi-k r)}
\end{aligned}
$$

$$
\begin{aligned}
E_{\phi}(r, \theta, \phi)= & \frac{1}{r} \sum_{m, n}\left[F_{m n} \frac{d}{d \theta} P_{n}^{|m|}(\cos \theta)\right. \\
& \left.-j \eta \frac{m}{\sin \theta} A_{m n} P_{n}^{|m|}(\cos \theta)\right](j)^{n+1} e^{j(m \phi-k r)}
\end{aligned}
$$

$$
\begin{gathered}
A_{m n}=\frac{\frac{j \omega \varepsilon r_{0}}{2 \pi k} \int_{\phi=0}^{2 \pi} \int_{\theta=0}^{\pi}\left(\left[E_{\theta} \frac{d}{d \theta} P_{n}^{|m|}-E_{\phi} \frac{j m}{\sin \theta} P_{n}^{|m|}\right] e^{-j m \phi}\right) \sin \theta d \theta d \phi}{\hat{H}_{n}^{(2) !} \frac{2}{2 n+1} \frac{(n+|m|) !}{(n-|m|) !} n(n+1)} \\
F_{m n}=\frac{\frac{r_{0}}{2 \pi} \int_{\phi=0}^{2 \pi} \int_{\theta=0}^{\pi}\left(\left[E_{\theta} \frac{j m}{\sin \theta} P_{n}^{|m|}+E_{\phi} \frac{d}{d \theta} P_{n}^{|m|}\right] e^{-j m \phi}\right) \sin \theta d \theta d \phi}{\hat{H}_{n}^{(2)} \frac{2}{2 n+1} \frac{(n+|m|) !}{(n-|m|) !} n(n+1)}
\end{gathered}
$$




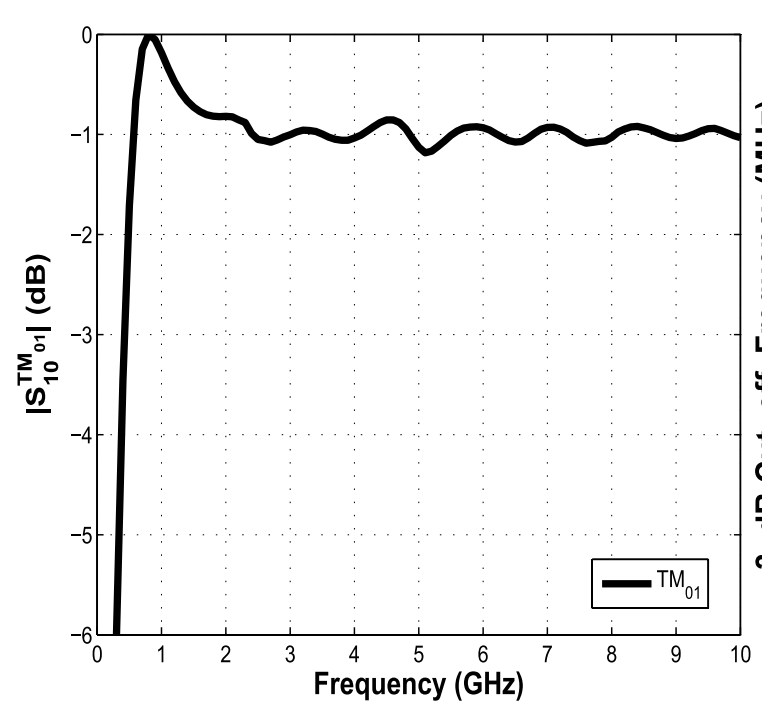

(a)

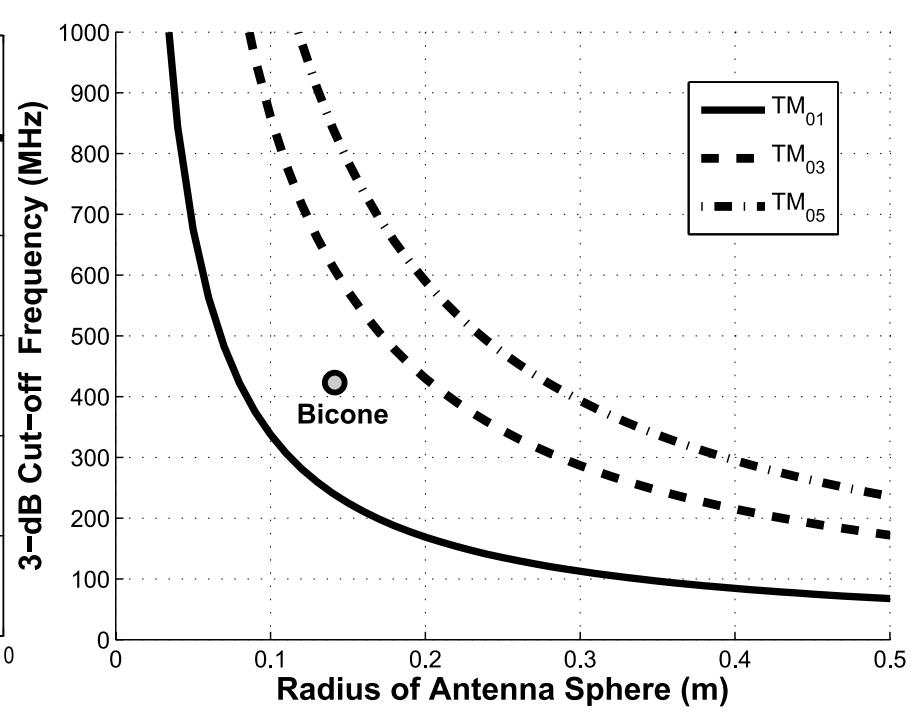

(b)

Figure 5. Bicone antenna performance: (a) $\left|s_{10}^{T M_{01}}\right|$ of $\mathrm{TM}_{01}$ mode and (b) fundamental limit $3 \mathrm{~dB}$ cutoff frequency versus antenna size for various spherical $\mathrm{TM}_{0 \mathrm{n}}$ modes and an example bicone (used by permission, T. Yang, 2008).

$$
\begin{aligned}
H_{\theta}(r, \theta, \phi)= & \frac{1}{r} \sum_{m, n}\left[j \frac{m}{\sin \theta} A_{m n} P_{n}^{|m|}(\cos \theta)\right. \\
& \left.-\frac{1}{\eta} F_{m n} \frac{\partial}{\partial \theta} P_{n}^{|m|}(\cos \theta)\right](j)^{n+1} e^{j(m \phi-k r)}(11) \\
H_{\phi}(r, \theta, \phi)= & \frac{1}{r} \sum_{m, n}\left[-A_{m n} \frac{\partial}{\partial \theta} P_{n}^{|m|}(\cos \theta)\right. \\
& \left.-j \frac{1}{\eta} \frac{m}{\sin \theta} F_{m n} P_{n}^{|m|}(\cos \theta)\right](j)^{n+1} e^{j(m \phi-k r)}
\end{aligned}
$$

Finally, the radiated power of each mode becomes

$$
\begin{aligned}
& P_{\text {rad }}^{T E_{m n}}=\pi \sum_{n=0}^{\infty} \sum_{m=-n}^{n} \frac{2 n(n+1)}{2 n+1} \frac{(n+|m|) !}{(n-|m|) !}\left(\frac{1}{\eta}\left|F_{m n}\right|^{2}\right) \\
& P_{\text {rad }}^{T M_{m n}}=\pi \sum_{n=0}^{\infty} \sum_{m=-n}^{n} \frac{2 n(n+1)}{2 n+1} \frac{(n+|m|) !}{(n-|m|) !}\left(\eta\left|A_{m n}\right|^{2}\right)
\end{aligned}
$$

The results of the spherical mode expansion for the bicone antenna of Figure $3 \mathrm{a}$ are shown in Figure $4 \mathrm{~b}$. The spherical $\mathrm{TM}_{01}$ mode dominants the entire frequency range and the lower bound of operational bandwidth is determined by the spherical $\mathrm{TM}_{01}$ mode. This observa- tion suggests that the size of ultrawideband antennas is limited by the excited fundamental spherical mode.

[18] Using equations (13) and (14), the power transfer from the antenna terminal and the spherical mode can be easily found from the antenna radiation model in Figure 4a. For example, the power transfer from antenna terminal to spherical $\mathrm{TM}_{0 \mathrm{n}}$ modes is given by

$$
\left|s_{n 0}^{T M_{0 n}}\right|^{2}=\frac{P_{r a d}^{T M_{0 n}}}{P_{i n c}}
$$

where $P_{r a d}^{T M_{0 n}}$ is the modal power and $P_{\text {inc }}$ is the incident power on the antenna. The computed $\left|s_{10}^{T M_{01}}\right|^{2}$ of the bicone antenna is plotted in Figure 5a.

[19] The size of antenna sphere versus 3-dB cutoff frequency for the various spherical TM modes is plotted in Figure 5b. As the antenna size decreases or the excited mode number increases, the cutoff frequency increases. Below the cutoff frequency, the input impedance of the TM-mode antenna becomes capacitive and impedance matching must be considered, as we addressed earlier. In practice, a wideband impedance match is not easily obtained. Loss can also be added to the antenna to obtain an acceptable return loss in this frequency range, but with reduced radiation efficiency and gain.

[20] The radiation performance of the bicone antenna as a function of size is best represented with the lower frequency cutoff (3-dB gain reduction) versus size, because the antenna is essentially unlimited on the upper frequency. When the 3-dB cutoff frequency of the bicone antenna is compared with the $3-\mathrm{dB}$ cutoff frequency for 
the spherical $\mathrm{TM}_{01}$ mode, it is apparent that it might be possible to design an antenna with a size below that of the bicone without sacrificing radiation efficiency. However, this reduction offers a major challenge for an ultrawideband antenna that avoids the trade-off in size, impedance bandwidth, and gain.

\section{Conclusions}

[21] Classical fundamental-limit theory on antenna size was interpreted from an ultrawideband antenna perspective. The frequency response of Chu's equivalent model for spherical TM modes suggests the concept of an ideal antenna, with the entire frequency response described by only two complex poles and an entire function. It was shown that the ideal antenna naturally has ultrawideband characteristics. The size limitations of ultrawideband antennas in terms of pulse width and $3-\mathrm{dB}$ cutoff frequency were developed and its usefulness was demonstrated with a bicone antenna. The 3 -dB cutoff frequency criterion can also be used to determine which antenna is more suitable in a frequency range of interest, either resonant or ultrawideband antennas. The demonstrated concepts and approaches in this paper are not limited to spherical $\mathrm{TM}_{0 \mathrm{n}}$ modes and can be generalized for $\mathrm{TM}_{\mathrm{mn}}$ and $\mathrm{TE}_{\mathrm{mn}}$ cases.

\section{References}

Baum, C. E., E. G. Farr, and D. V. Giri (1999), Review of impulse-radiating antennas, in Review of Radio Science 1996-1999, Wiley-IEEE Press, Hoboken, N. J.

Chu, L. J. (1948), Physical limitations on omni-directional antennas, J. Appl. Phys., 19, 1163-1175.

Collin, R. E., and S. Rothschild (1964), Evaluation of antenna Q, IEEE Trans. Antennas Propag., 12, 23-27.

Davis, W. A. (2005), Review of fundamental limits of antennas and identification of the terms missing in recent developments, paper presented at USNC/URSI National Radio Science Meeting, Boulder, Colo.
Davis, W. A., T. Yang, and W. L. Stutman (2007), A new fundamental limit for omni-directional antennas, paper presented at CNC/USNC North America Radio Science Meeting, Ottawa, Ont., Canada.

Fano, R. M. (1950), Theoretical limitations on the broadband matching of arbitrary impedances, J. Franklin Inst., 249, $57-154$.

FEKO (2007), Suite version 5.3, EM Software and Syst., Hampton, Va. (Information available at http://www.feko.info).

Grimes, D. M., and C. A. Grimes (1999), Radiation $Q$ of dipole generated fields, Radio Sci., 34, 282-296.

Gustafsson, M., and S. Nordebo (2006), Bandwidth, Q factor, and resonance models of antennas, Prog. Electromagn. Res., $62,1-20$.

Hansen, J. E. (1988), Spherical Near-Field Antenna Measurements, Electromagn. Wave Ser., Inst. of Electr. Eng., London.

Licul, S. (2004), Ultra-wideband antenna characterization and modeling, doctoral dissertation, Va. Polytech. Inst. and State Univ., Blacksburg.

McLean, J. S. (1996), A reexamination of the fundamental limits on the radiation $Q$ of electrically small antennas, IEEE Trans. Antennas Propag., 44, 672-675.

Stutzman, W. L., and G. A. Thiele (1998), Antenna Theory and Design, 2nd ed., 648 pp., John Wiley, New York.

Villalobos, M. C., H. D. Foltz, J. S. McLean, and I. S. Gupta (2007), Broadband tuning limits on UWB antennas based on Fano's formulation, in Ultra Wideband Short Pulse Electromagnetics, vol. 8, edited by C. E. Baum, A. P. Stone, and J. S. Tyo, pp. 83-88, Springer, New York.

Wheeler, H. A. (1947), Fundamental limitations of small antennas, Proc. IRE, 35, 1479-1484.

W. A. Davis, W. L. Stutzman, and T. Yang, Virginia Tech Antenna Group, Department of Electrical and Computer Engineering, Virginia Polytechnic Institute and State University, 302 Whittemore Hall, 0111, Blacksburg, VA 24061, USA. (wadavis@vt.edu) 